\title{
Reversible Tuning of the Plasmoelectric Effect in Noble Metal Nanostructures Through Manipulation of Organic Ligand Energy Levels
}

\author{
Thakshila Liyanage, ${ }^{1}$ Malpuri Nagaraju, ${ }^{1}$ Merrell Johnson, ${ }^{2}$ Barry B. Muhoberac, ${ }^{1}$ and \\ Rajesh Sardar*,1
}

${ }^{1}$ Department of Chemistry and Chemical Biology, Indiana University-Purdue University Indianapolis, 402 N. Blackford Street, LD326, Indianapolis, Indiana, 46202, United

\section{States}

2Department of Physics, Purdue University Fort Wayne, 2101 E. Coliseum Blvd.

Fort Wayne, Indiana, 46805, United States

Keywords: metal nanostructures, gold triangular nanoprisms, localized surface plasmon resonance, plasmon electron density, plasmoelectric effect, work function, spectral reversibility

\section{Table of Content (TOC) Graphic}
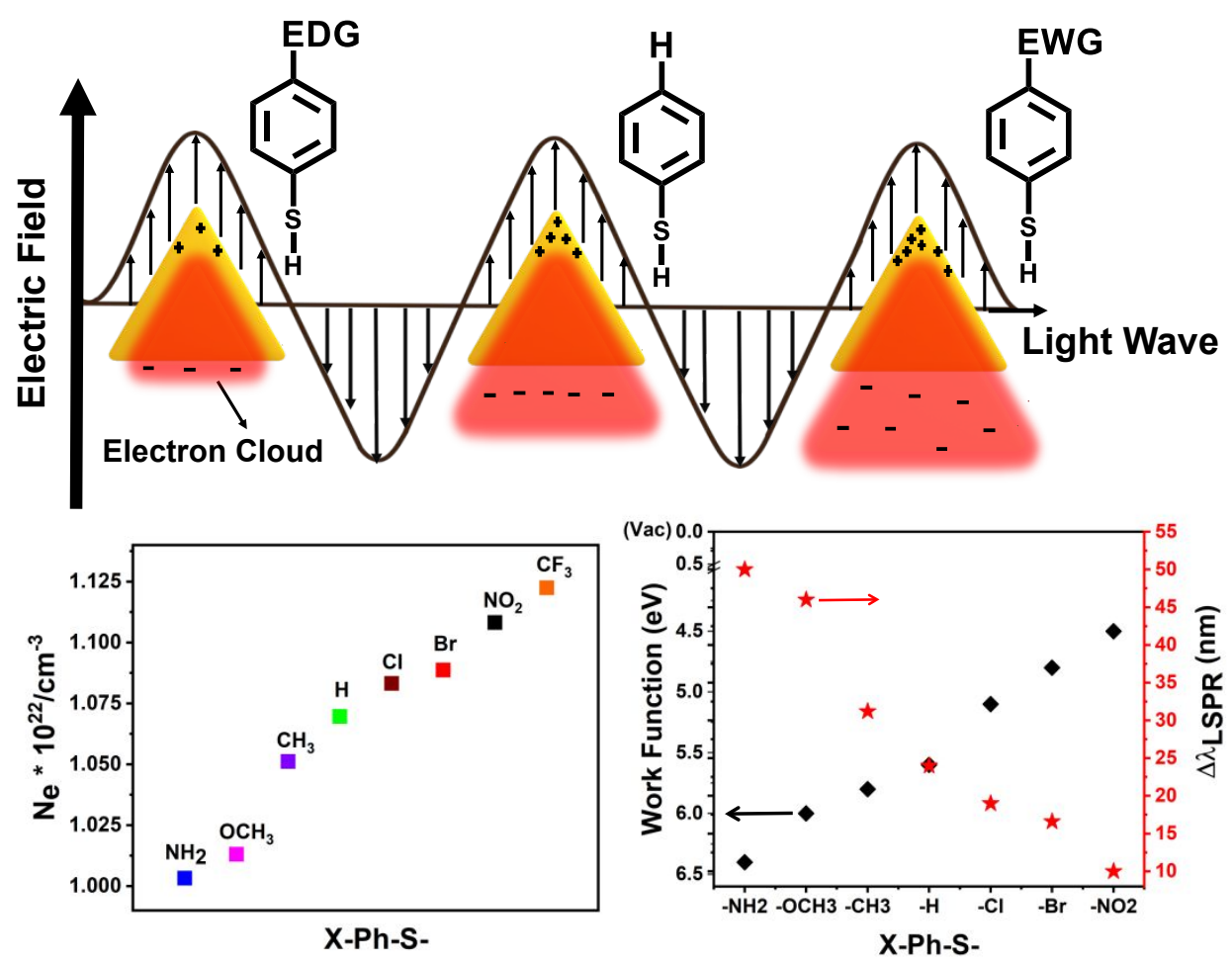


\begin{abstract}
:
Ligand-controlled tuning of localized surface plasmon resonance (LSPR) properties of noble metal nanostructures is fundamentally important for various optoelectronic applications such as photocatalysis, photovoltaics, and sensing. Here we demonstrate that the free carrier concentration of gold triangular nanoprisms ( $\mathrm{Au}$ TNPs) can be tuned up to $12 \%$ upon functionalization of their surface with different para-substituted thiophenolate (X-Ph-S-) ligands. We achieve this unprecedentedly large optical response (plasmoelectric effect) in TNPs through the selective manipulation of electronic processes at the Au-thiolate interface. Interestingly, thiophenolates with electron withdrawing(donating) groups $(X)$ produce $\lambda_{\text {LSPR }}$ blue(red) shifts with broadening(narrowing) of localized surface plasmon resonance peak $\left(\lambda_{\text {LSPR }}\right)$ linewidths. Surprisingly, these experimental results are opposite to a straightforward application of the Drude model. Utilizing density functional theory calculations, we develop here a frontier molecular orbital (MO) approach of Au-thiophenolate interactions in the solid-state to delineate the observed spectral response. Importantly, all the spectroscopic properties are fully reversible by exchanging thiophenolates containing electron withdrawing groups with thiophenolates having electron donating groups, and vice versa. Based on the experimental data and calculations, we propose that the delocalization of electrons wave function controls the free carrier concentration of $\mathrm{Au}$ and thus, the LSPR properties rather than simple electronic properties (inductive and/or resonance effects) of thiophenolates. This is further supported by the experimentally determined work functions, which are tunable over $1.9 \mathrm{eV}$ in the X-Ph-Spassivated Au TNPs. We believe that our unexpected finding has great potential to guide in developing unique noble metal nanostructure-organic ligand hybrid nanoconjugates, which could allow us to bypass the complications associated with off-resonance LSPR activation of noble metal-doped semiconductor nanocrystals for various surface plasmon-driven applications.
\end{abstract}


Upon illumination of visible light onto noble metal nanostructures, the incident electric field triggers coherent oscillation of conduction electrons ("surface plasmon excitations") of the nanostructures. This nanoscale optical phenomenon is known as localized surface plasmon resonance (LSPR). ${ }^{1,2}$ Traditionally, LSPR properties are controlled by changing the size, shape, and local dielectric environment of the nanostructures. ${ }^{3-5}$ According to the Drude model (Eq. 1 and 2), charging of a nanostructure will alter its free carrier concentrations - an increase (decrease) in electron density of the plasmonic nanostructure, the bulk plasmon frequency $\left(\omega_{p}\right)$ is expected to increase (decrease), and therefore the wavelength $(\lambda)$ to decrease (increase), which results in a blue (red)-shift of the LSPR peak $\left(\lambda_{\mathrm{LSPR}}\right)$ position - should result in shifting of surface plasmon resonance frequency. ${ }^{6}$ Here, $N_{e}$ is the density of free electrons in the metal, $m$ is the effective mass of the electron and $\varepsilon_{0}$ is the dielectric function. Therefore, noble metal nanostructures can be considered as "plasmoelectronic materials" 7 in which precise tuning of the $\lambda_{\mathrm{LSPR}}$ position is highly plausible by external electric fields when nanostructures are in contact with conductive surfaces or have light irradiation.

$$
\begin{aligned}
& \omega_{p}^{2}=\frac{N_{e} e^{2}}{m \epsilon_{0}} \\
& \omega_{p} \propto \frac{1}{\lambda}
\end{aligned}
$$

Recently, Sheldon et al. ${ }^{8}$ experimentally demonstrated a reversible shift of $\lambda_{\mathrm{LSPR}}$ position of $\mathrm{Ag}$ nanostructures by changing the $\mathrm{N}_{\mathrm{e}}$ in the metal through external electrostatic fields, $a$ plasmoelectric effect. Hoener et al. reported the LSPR response of anisotropic Au nanostructures due to capacitive charging. ${ }^{9}$ Additionally, Moskovits and coworkers showed photo-induced shifts of the $\lambda_{\mathrm{LSPR}}$ positions of Au nanostructures contacting with a thin layer of conducting metal-oxides. ${ }^{10}$ All these studies validate the Drude model as described in Eq. 1 and 2. In this context, one could envision plasmoelectric effects upon attachment of organic ligands to nanostructures that are capable of altering their free carrier concentrations positively/negatively by withdrawing/injecting charges through inductive or resonance effects. Importantly, the $\lambda_{\mathrm{LSPR}}$ position is expected to be reversibly cyclable upon replacing charge donating ligands with charge withdrawing ligands and vice versa. To our knowledge, such ligand-controlled, fully reversible tuning of plasmoelectric effects in metal nanostructures has yet to be demonstrated but is important for the preparation of novel, inorganic-organic hybrid nanostructures for optoelectronic applications. 
In this letter, we show how covalently attached, para-substituted thiophenolate ligands (X-Ph-S-; $\mathrm{X}=-\mathrm{NH}_{2},-\mathrm{OCH}_{3},-\mathrm{CH}_{3},-\mathrm{H},-\mathrm{Cl},-\mathrm{Br},-\mathrm{NO}_{2}$, and $-\mathrm{CF}_{3}$ ) that varying their electron donation/withdrawal ability alter the TNP LSPR properties ("a plasmoelectric effect") of chemically synthesized gold triangular nanoprisms (Au TNPs) in solid-state and how the energy level alignment between TNPs and ligand molecular orbitals (MOs) of the X-Ph-SH series control the $\mathrm{N}_{\mathrm{e}}$ of Au TNPs up to $12 \%$, for the first time. We observe fully reversible tuning of the $\lambda_{\text {LSPR }}$ position of Au TNPs for at least five cycles upon passivation with X-Ph-S- ligands.

Surprisingly, $\mathrm{X}-\mathrm{Ph}-\mathrm{S}$ - ligands containing strong electron withdrawing $-\mathrm{CF}_{3}$ and strong electron donating $-\mathrm{NH}_{2}$ groups produce blue- and red-shifts of the $\lambda_{\mathrm{LSPR}}$ position, respectively, with respect to thiophenolate (H-Ph-S-). Our experimental results and proposed MO theory of the TNP-S-Ph-X system show that the opposite LSPR response in comparison to the Drude model (Eqs. 1 and 2), is not controlled by the change in local dielectric environment of the Au TNPs or variation in the number of X-Ph-S-/TNP $\left(\mathrm{X}-\mathrm{Ph}-\mathrm{S}-/ \mathrm{nm}^{2}\right)$. It is, perhaps, delocalization of electron wave functions either from or to hybrid orbitals that are formed between electronic states of $\mathrm{Au}$ TNPs and MOs of thiophenol. Delocalization occurs because of the appropriate alignment of MO energy levels, alters the overall free carrier concentration of the TNP and consequently the LSPR properties. Finally, between electron donating and withdrawing groups on X-Ph-S-, we determine an $\sim 1.9 \mathrm{eV}$ change in the work function $(\phi)$ of Au TNPs which supports the proposed electron wave functions delocalization mechanism. Together, such an unprecedentedly large range of ligand-controlled optical spectral tunability of noble metal nanostructures in the visible region of the solar spectrum should be beneficial for development of unique electronic materials for plasmon-driven solar cells ${ }^{11-13}$ and photocatalytic ${ }^{14,15}$ applications, while circumventing the difficulties of off-resonance LSPR activation of noble metal-doped semiconductor nanocrystals. ${ }^{8}$, 16

We selected Au TNPs to investigate ligand-controlled reversible tunability of plasmoelectric effects in noble metal nanostructures because their atomically flat surface should allow the formation of a well-controlled self-assembled monolayer of X-Ph-S- ligands. Moreover, the TNPS-Ph-X system has several important nanoscale structural properties that are found such as (1) strong electromagnetic (EM) field enhancement at their sharp tips and edges that should induce a large LSPR response, ${ }^{17-19}$ (2) sharp structural features of TNPs that are expected to facilitate strong Au-S-Ph-X interactions at the metal-ligand interface, ${ }^{20}$ and (3) high stability of the Au-S bond due to soft-soft covalent interactions. These properties allow spectroscopic characterization that is critical for device applications in the solid-state. ${ }^{19,} 21,22$ As shown in 
Figure 1A, we investigated the LSPR response of Au TNPs by attaching them onto a silanized glass substrate followed by ligand exchange reactions with $\mathrm{X}-\mathrm{Ph}-\mathrm{S}-; \mathrm{X}=-\mathrm{NH}_{2},-\mathrm{OCH}_{3},-\mathrm{CH}_{3},-\mathrm{H}$, $-\mathrm{Cl},-\mathrm{Br},-\mathrm{NO}_{2}$, and $-\mathrm{CF}_{3}$ in the solid-state. Figure 1B and Figure $\mathrm{S} 1$ represent atomic force (AFM) and scanning electron (SEM) microscopy images of trioctylamine (TOA)-passivated, Au TNPs with an average $42 \mathrm{~nm}$ edge-length and $8.5 \mathrm{~nm}$ height that display an LSPR dipole peak $\left(\lambda_{\mathrm{LSPR}}\right)$ at $775 \mathrm{~nm}$ in air (Figure S2).

Ligand exchange by thiophenol produces fully X-Ph-S- ligand-passivated TNPs as confirmed by surface-enhanced Raman scattering (SERS) measurements in which disappearance of the C-N Raman stretch at $1035 \mathrm{~cm}^{-1}$ and appearance of the C-S and aromatic C=C stretches at 1083 and $1573 \mathrm{~cm}^{-1}$, respectively, are observed (Figure S3). As illustrated in Figure 1C and D, an $\sim 24 \mathrm{~nm}$ red-shift in the $\lambda_{\mathrm{LSPR}}$ of TNPs is observed that could be due to the change in the local dielectric environment around the TNPs. Passivation of Au TNPs with X-Ph-S- ligands containing strong electron withdrawing $\left(X=-\mathrm{CF}_{3}\right)$ and strong electron donating $\left(\mathrm{X}=-\mathrm{NH}_{2}\right)$ groups produces $\sim 20$ and $\sim 25 \mathrm{~nm}$ blue and red shifts of the $\lambda_{\text {LSPR }}$ position, respectively, with respect to H-Ph-S- (see Figure 1C). Figure 1D and Figure S4A show the observed $\Delta \lambda_{\text {LSPR }}\left(\lambda_{X}\right.$ Ph-S-passivated $-\lambda_{\text {TOA-passivated }}$ ) upon ligand exchange of TOA-passivated Au TNPs with X-Ph-SH. Interestingly, the magnitude of the LSPR red and blue shifts follows the increasing order of electron donation $\left(-\mathrm{CH}_{3}>-\mathrm{OCH}_{3}>-\mathrm{NH}_{2}\right)$ and electron withdrawal $\left(-\mathrm{Cl}>-\mathrm{Br}>-\mathrm{NO}_{2}>-\mathrm{CF}_{3}\right)$ abilities of the para-substitutions, respectively. The change in the $\lambda_{\mathrm{LSPR}}$ position ( $\Delta \square_{\mathrm{LSPR}}$ in $\mathrm{nm}$ or meV) from fully TOA-passivated TNPs upon ligand exchange with X-Ph-SH for the different substitutions $(X)$ does not follow the refractive index trend (see Figure 1E and Figure S4B). If so one would expect nearly identical $\Delta \lambda_{\mathrm{LSPR}}$ values for $-\mathrm{Br}$ and $-\mathrm{NH}_{2}$ substitutions, whereas $-\mathrm{CH}_{3}$ and $-\mathrm{OCH}_{3}$ substituted X-Ph-S- ligands should provide lesser shifts than either $\mathrm{Cl}$ or $\mathrm{Br}$ substitutions. As shown in Table $\mathrm{S} 1$, the difference in refractive indices between ligands is relatively small $(\sim 0.1)$ but it is still significant enough to observe large LSPR shifts with the appropriate trend in which LSPR peak should red-shift with an increase in refractive index for $\sim 42 \mathrm{~nm}$ edge-length and $8.5 \mathrm{~nm}$ thick Au TNPs. ${ }^{23}$ However, we have not taken into account that the refractive index of surface passivating ligand would be different when they are attached onto the surface of Au TNPs with a particular orientation than the ensemble measurements in the free state with all molecular orientations. It is possible that the trend of refractive index data tabulated in Table S1 would remain same. Nevertheless, in a very simplistic LSPR phenomenon, the trend in $\Delta \boldsymbol{\lambda}_{\mathrm{LSPR}}$ values with respect to the electronic character of parasubstitutions is somewhat surprising because according to the Drude model $\omega_{p}$ is expected to 
increase (decrease) $\left[\lambda_{\mathrm{LSPR}}\right.$ blue shift (red) shift] for X-Ph-S- ligands containing the electrondonating (-withdrawing) groups [e.g., $\mathrm{X}=-\mathrm{NH}_{2}\left(-\mathrm{CF}_{3}\right.$ ], as described by Eq. 1 and 2 (i.e., the Drude model).

Beside refractive index-related effects, as described above, two other factors, i.e., the variation in the number of X-Ph-S- ligands attached per TNP (surface coverage) and the dipole moment of TNP-attached ligands would also influence the LSPR properties including the $\lambda_{\text {LSPR }}$ position of our metallic nanostructures. We hypothesize that the attachment of X-Ph-S- ligands onto $\mathrm{Au}$ TNPs produces unexpected metal-ligand electronic binding properties because of the particular aspects of orbital alignment and overlap allowing delocalization of plasmonic electron wave functions, thus resulting in the changes in the $\mathrm{N}_{\mathrm{e}}$ of TNPs and the unexpected $\lambda_{\text {LSPR }}$ response, see Figure 2 (vide infra). Before we present our proposed MO theory in order to explain the usual spectral response of X-Ph-S-passivated Au TNPs, we must acknowledge that the electrostatic interaction between TNPs and the oriented dipole moment of passivating ligands could alter the surface density of conduction electron that results in variation in the localized dipole moment of TNPs, and thus their LSPR responses. In order to precisely determine the role of ligands' dipole moments on the LSPR properties of TNPs, it is critical to determine the different structural models such as ligand-ligand and inter-TNPs interactions because these interactions could influence the overall dipole moment of the ligand-passivated Au TNPs. Quantitative determination of these models require sophisticated theoretical calculations that are beyond the scope of this letter. Later part in this letter, we discussed about the surface coverage and the resulting LSPR properties of X-Ph-S-passivated Au TNPs. 

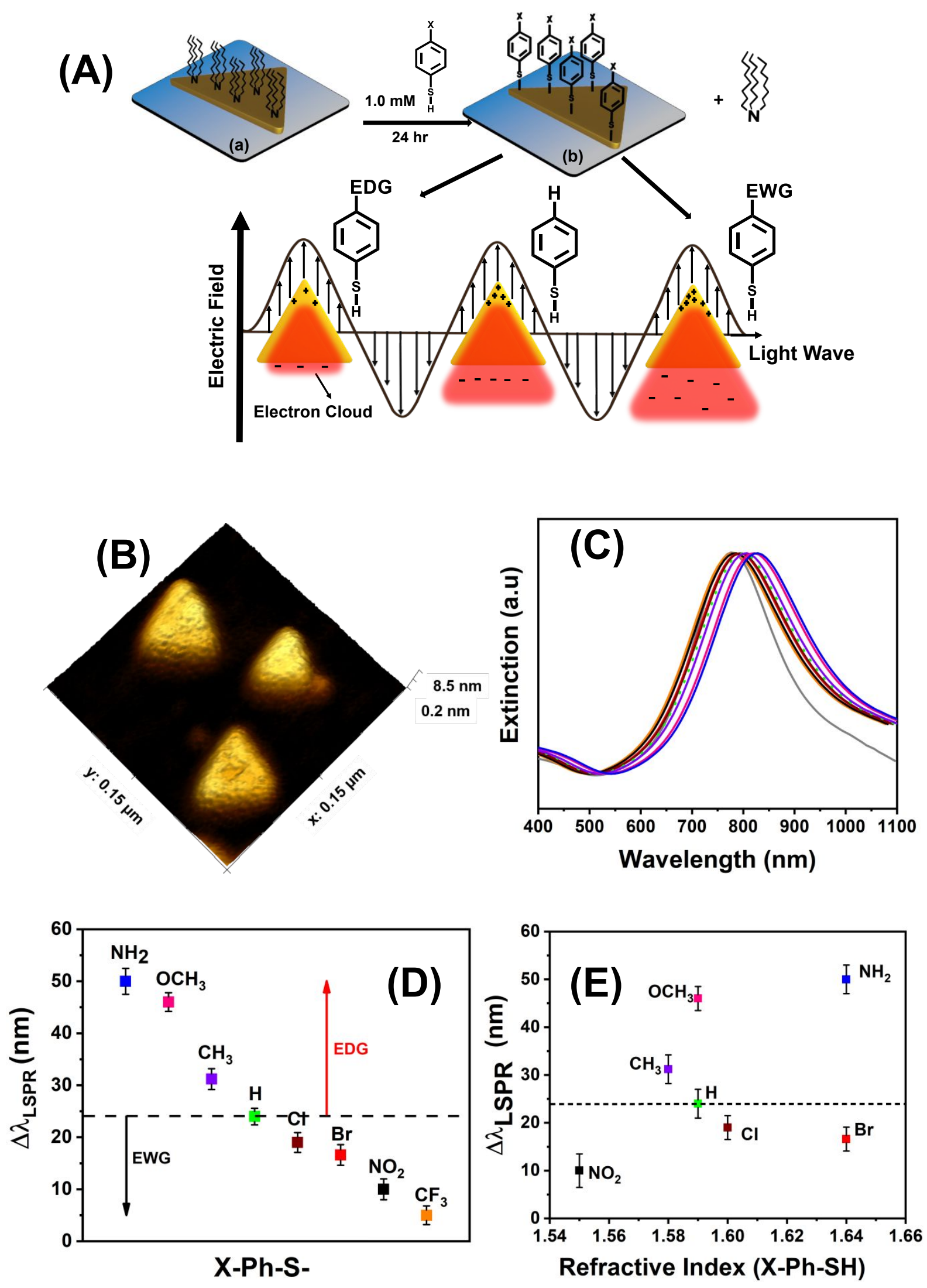
Figure 1. Structural and optical characterizations of different X-Ph-S-passivated Au TNPs in the solid-state. (A) Starting with TOA-passivated Au TNPs, TOA can be replaced by different thiophenolate ligands containing electron donating (EDG) and withdrawing (EWG) groups. An oscillating electric field then interacts differently with the TNP. Note that, the metal with the EWG has a higher charge density and conversely with the EDG, it is lower. The image is not to scale. (B) AFM image of the TOA-passivated Au TNPs. (C) Experimentally determined LSPR spectra of para-substituted thiophenolate (X-Ph-S-)-passivated Au TNPs: TOA- (gray-774 $\mathrm{nm}$ ), and $X-P h-S$ - passivated: $X=\mathrm{CF}_{3}$ (orange-779 nm ), $X=\mathrm{NO}_{2}$ (black-784 nm), $X=\mathrm{Br}$ (red$791 \mathrm{~nm}), X=\mathrm{Cl}$ ( wine-793 nm), $X=H$ (green dotted line-798 nm), $X=\mathrm{CH}_{3}$ (Purple-805 nm), $X$ $=\mathrm{OCH}_{3}\left(\right.$ pink-820 nm), $X=\mathrm{NH}_{2}\left(\right.$ Blue-824 nm). (D) Summary of the observed $\Delta \lambda_{L S P R}\left(\lambda_{X-P h-S-}\right.$ passivated $-\lambda_{\text {TOA-passivated }}$ Au TNPs) upon passivation of Au TNPs with X-Ph-S- through the ligand exchange chemistry. Red arrow indicates the $\lambda_{L S P R}$ shifts after passivation with various electron donating groups (EDGs) and black arrow indicates the $\lambda_{L S P R}$ shifts after passivation with electron withdrawing groups (EWGs) compared to when Au TNPs were functionalized with $H$ Ph-S-. (E) Figure summarizes the $\triangle \lambda_{L S P R}$ changes upon passivation of Au TNPs with X-Ph-Svs. refractive index of $X-P h-S$ - ligands.

As reported in the literature for Au nanostructures ${ }^{24}$ and according to $\mathrm{MO}$ theory, ${ }^{25}$ chemical attachment or adsorption of organic molecules creates new hybrid orbitals. To address the unexpected LSPR shifts we took advantage of the MO theory because it has a unique potential to appropriately describe the metal-ligand interaction in solid-state from a thermodynamic view point. Furthermore, MO theory can explain the change in work function $(\phi)$ of metal upon covalent bond formation with ligands. We predict that in the case of $\mathrm{X}-\mathrm{Ph}$-S- ligands containing electron-donating groups (e.g., $-\mathrm{NH}_{2},-\mathrm{CH}_{3}$, etc.) completely empty hybrid bonding (HOMO') and anti-bonding (LUMO') orbitals are formed when two empty orbitals interact, here considering $\mathrm{Au}$ has continuum of energetic states (LUMOs) and the ligand contains LUMOs (Figure 2A). ${ }^{25}$ Interactions between two empty orbitals would be discounted, however, moving electron densities from the Au Fermi level to empty HOMO' is considered to be stabilizing and attractive and that would together result in Au-S-Ph-X bonding. When illuminated with visible light during the UV-visible spectroscopic characterization, the LSPR active low energy electrons ("plasmonic electrons") near the Fermi level are generated. There are two possibilities involving these 
electrons: (1) Wave functions of plasmonic electrons could delocalize into empty HOMO', which reside below the Au Fermi level $\left(E_{F},-5.5 \mathrm{eV}\right) ; 26$ (2) Plasmonic electrons are being transferred to empty HOMO'. In our system we believe that the plasmonic electron wave functions are delocalizing, because if the electrons are transferred from Au-to-HOMO' then excess positive charge (residual holes) will build up that can slowly destroy the TNPs and permanently change the LSPR properties from conducting the measurements in $\mathrm{N}_{2}$ filled cuvette where no potential hole scavengers were available. Delocalization of electron wave functions decreases the overall $N_{e}$ of $A u$ TNPs, which supports the red shift of the $\lambda_{\text {LSPR }}$ position. In contrast, as shown in Figure 2B, an interaction between X-Ph-S- ligands (containing electron-withdrawing groups e.g., $-\mathrm{NO}_{2}$, $-\mathrm{CF}_{3}$, etc.) and $\mathrm{Au}$ is a four-electron and two-orbital system in which the LUMO' rises above the Au Fermi energy. Four electrons will occupy HOMO' and LUMO' making the interaction destabilizing and repulsive. However, extension of LUMO' electron wave functions to the Au Fermi level stabilizes the system and increases the $\mathrm{N}_{e}$ in TNPs. This electronic process leads to a blue shift of the $\lambda_{\text {LSPR }}$ position. We performed density functional theory (DFT) calculations on $\mathrm{NH}_{2}-\mathrm{Ph}-\mathrm{S}-\mathrm{Au}$ using a simple Au-thiolate "staple" structures $\left[\mathrm{Au}(\mathrm{X}-\mathrm{Ph}-\mathrm{S}-)_{2}\right]^{27-29}$ to find the $\mathrm{MO}$ energy levels of the of HOMO' and LUMO' and then found to be -5.850 and $-3.158 \mathrm{eV}$, respectively. Similarly, DFT calculations provided the HOMO' and LUMO' of $\mathrm{NO}_{2}-\mathrm{Ph}-\mathrm{S}-\mathrm{Au}$ at 6.911 and $-4.005 \mathrm{eV}$, respectively. Considering an $8 \mathrm{~nm}$ thick Au TNP as a plasmonic slab, the energy of the plasmonic electron would be $-3.28 \mathrm{eV} .{ }^{26}$ Under this calculation, delocalization of plasmonic electron wave function into the HOMO' orbital is thermodynamically favorable for $\mathrm{NH}_{2}-\mathrm{Ph}$-S- passivation (Figure 2A). Also, in order to stabilize the destabilize hybrid MOs, delocalization of the electron wave function from LUMO' to Au would be energetically favorable for the $\mathrm{NO}_{2}-\mathrm{Ph}-\mathrm{S}-\mathrm{Au}$ system (Figure 2B). This electronic process would lead to an increase in Au free carrier concentrations. Together, the slab theory and DFT calculations support the proposed MO diagram. Newly formed hybrid MOs contain the properties of both metal and ligands. The supporting Information file contains further information concerning the DFT calculations. It is also important to mention that in the formation of hybrid orbitals (HOMOs' and LUMOs') from mixing of individual orbitals of Au and X-Ph-S-, one must consider that the metalligand interactions depend upon the distance between the energy levels and their overlap and symmetry, such that different ligands will interact differently with the metal. The interactions depend on the appropriate energy level alignment between the MOs of metal and ligand and their coupling constants. Therefore, the better the energy level alignment and is the higher the coupling. The probability of the formation of a larger number of hybrid MOs is also higher which allows facile delocalization of plasmonic electron wave functions. Together, this electronic 
process strongly modulates the LSPR properties of ligand-passivated Au TNPs. Perhaps, multiple HOMOs and LUMOs could interact but for simplicity, here we only discuss only one set of MO interactions.
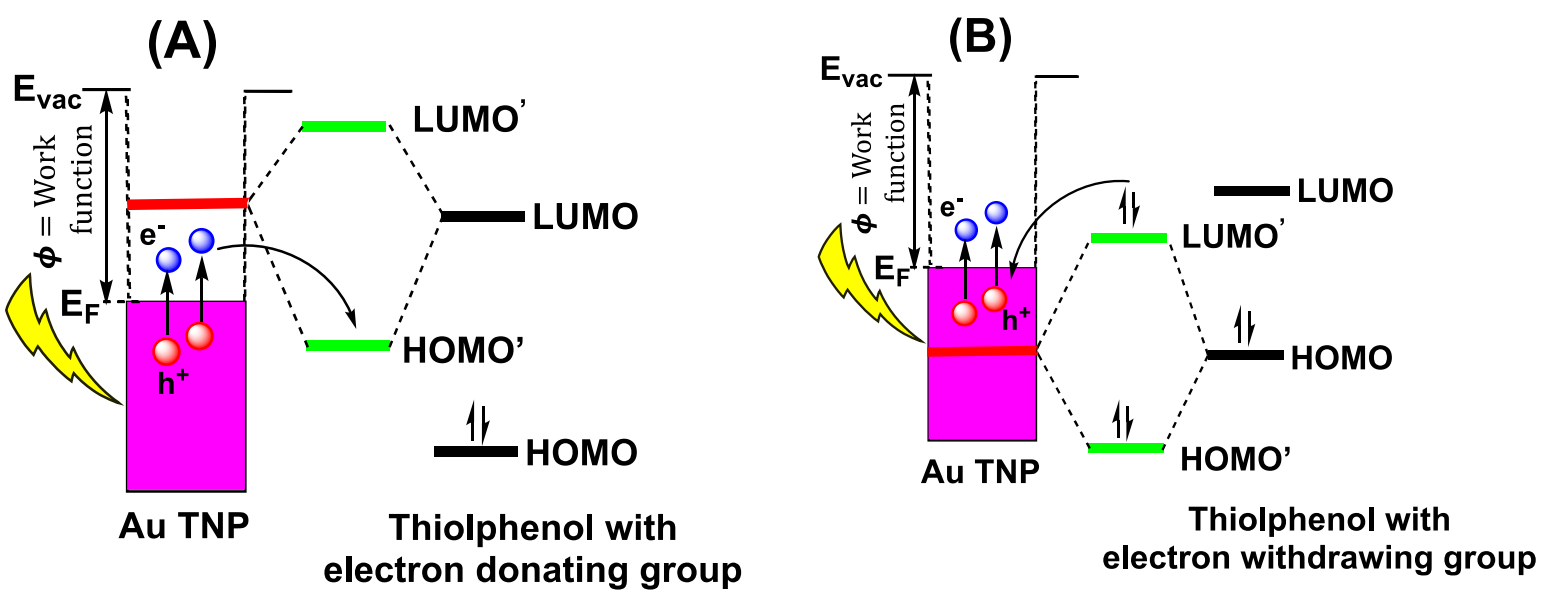

Figure 2. Proposed metal-ligand orbital binding scheme between Au TNP and parasubstituted, thiophenolate interfaces emphasizing the electron contributions to the Fermi level. (A) With the ligand LUMO orbital, the para-substituted thiophenolate with an electron donating group provides a better energy match to an unoccupied metal orbital resulting in a hybrid metal-ligand MO below the Fermi level and thus allowing delocalization of plasmonic electron wave functions from the metal to stabilize the destabilized hybrid MOs. $(B)$ With the ligand HOMO orbital, the substituted thiophenolate with an electron withdrawing group provides a better energy match to an occupied metal orbital resulting in a hybrid metal-ligand MO above the Fermi level and thus allowing delocalization of antibonding electron wave functions from the hybrid LUMO' to metal to stabilize the system and increases the $\mathrm{N}_{\mathrm{e}}$ in TNPs. Images are not to scale.

In order to support the proposed electron wave function delocalization-controlled modulation of the LSPR properties of Au TNPS, we carefully examined UV-visible extinction spectra of all the X-Ph-S-passivated TNPs. Figure 3A illustrates the relationship between fullwidth at half maxima (FWHM) and various para-substitutions in which $\mathrm{NH}_{2}-\mathrm{Ph}-\mathrm{S}$ - and $\mathrm{CF}_{3}-\mathrm{Ph}-\mathrm{S}$ passivated $\mathrm{Au}$ TNPs show an $\sim 8 \%$ decrease and $23 \%$ increase in the FWHM of the extinction spectra, respectively, in comparison to the FWHM of H-Ph-S-passivated Au TNPs. The values 
in eV for Figure 3A are presented in Figure S5. Our experimental spectral linewidth data are in agreement with literature suggesting that an increase in $\mathrm{N}_{\mathrm{e}}$ of a nanostructure leads to an increase in damping of the oscillation of the conduction electrons. ${ }^{9}, 30$ This electronic phenomenon is responsible for the broadening of the LSPR spectrum. ${ }^{30}$ As shown in Figure 3B, we calculated $\mathrm{N}_{\mathrm{e}}$ of Au TNPs upon passivation with X-Ph-S- utilizing Eqs. 3 and 4, as described in the literature. ${ }^{31}$ Clearly $\mathrm{NH}_{2}-\mathrm{Ph}-\mathrm{S}$ - and $\mathrm{CF}_{3}-\mathrm{Ph}-\mathrm{S}$-passivated $\mathrm{Au}$ TNPs show an $\sim 6.2 \%$ decrease and $5.2 \%$ increase in $\mathrm{N}_{\mathrm{e}}$, respectively, as compared to the $\mathrm{N}_{\mathrm{e}}$ of $\mathrm{H}$-Ph-S-passivated Au TNPs. Importantly, an $8 \mathrm{~nm}$ thick Au TNP as a plasmonic slab is capable of generating $\sim 10^{5}$ plasmonic electrons under illumination of incident light perpendicular to the TNP surface. ${ }^{26}$ Therefore, the change in electron density upon surface ligand passivation is in good agreement. We do not fully understand the reason underlying the relatively large change in FWHM of the extinction spectrum of $\mathrm{CF}_{3}$-Ph-S-passivated Au TNPs, which provides a smaller change in $\mathrm{N}_{e}$ compared to $\mathrm{NH}_{2}$-Ph-S-passivation, because one would expect that the larger the $\mathrm{N}_{\mathrm{e}}$ change, the larger FWHM value.

$\omega_{s p}=\sqrt{\frac{\omega_{p}^{2}}{1+2 \varepsilon_{m}}-\gamma^{2}}$

Here $\omega_{s p}$ is the surface plasmon frequency, and $\varepsilon_{\mathrm{m}}$ is the dielectric constant of plasmonic materials. The electrons of the metal oscillate due to the incident electromagnetic field, and the motion is collision damped with a bulk collision frequency $\gamma$. Metals have free electrons in their conduction band, therefore large frequencies in the visible and near visible region lead to negligible damping frequency, and this approximation leads to modification of the Drude model where damped oscillator frequency (Eq. 3) can be rewritten as Eq. 4. We obtained the $\omega_{s p}$ value for different X-Ph-S-passivated Au TNPs utilizing the $\lambda_{\text {LSPR }}$ peak maxima as shown in the UVvisible extinction spectra (Figure 1C).

$$
\omega_{s p}=\sqrt{\frac{\omega_{p}^{2}}{1+2 \varepsilon_{m}}}
$$


It is also important to note that in the $N_{e}$ calculations, we considered $\Delta \lambda_{\mathrm{LSPR}}$ as induced only by nanostructure plasmoelectric effects and not due to the changes in the local refractive index by individual X-Ph-S- ligands and/or the induced dipole of the metal-ligand system. Including individual components to fully quantify both $\mathrm{N}_{\mathrm{e}}$, dipole moment and refractive index effects on $\Delta \lambda_{\text {LSPR }}$ values of Au TNPs upon surface modification with various X-Ph-S- ligands require sophisticated theoretical calculations, which are beyond our expertise. We should also mention that the change in FWHM of metallic nanostructures due to charging and discharging is somewhat controversial. During the preparation of our manuscript, Lee et al. ${ }^{20}$ reported the chemical interface damping (CID) phenomenon ${ }^{16,30,32}$ - direct energetic electron transfer from plasmonic metals to their surface-bound, strongly interacting passivating ligands that causes damping in a homogenous LSPR linewidth - in thiolate ligand-passivated gold bipyramids. Here the authors observed a weak FWHM change of $\sim 20$ and $\sim 15 \mathrm{meV}$ for $\mathrm{NO}_{2}$-Ph-S- (electron withdrawing) and $\mathrm{NH}_{2}$-Ph-S- (electron donating), respectively, that is also opposite to the Drude model. An 20-fold higher FWHM value is observed for Au TNP-passivated with $\mathrm{NH}_{2}-\mathrm{Ph}-\mathrm{S}$ - in comparison to work reported for gold bipyramids for the identical ligand. Two possible reasons could lead to such an unprecedentedly large FHWM value observed in our system: (1) Atomically flat surfaces of Au TNPs should allow the formation of a better self-assembled monolayer of X-Ph-S- as compared to bipyramids. Therefore, more hybrid MOs are expected to form that would lead to a better delocalization of the electron wave function. (2) The LSPR characterizations of X-Ph-S-passivated bipyramids were conducted at a single nanoparticle level that resulted in an inherently narrow LSPR peak. In contrast, we characterized our LSPR responses through ensemble measurements, which always provide broad LSPR peak and high FWHM value due to heterogeneity of Au TNP size. We are actively investigating the LSPR properties of different X-Ph-S-passivated Au TNPs at a single nanoparticle level. Nevertheless, the literature work lacks a proper ligand reference (e.g., H-Ph-S-) to fully rationalize the CID effect along with having a very limited selection of X-Ph-S- ligands. Moreover, CID should induce excess positive charge ("hot-hole") accumulation within the occupied states of a noble metal, and without the use of hole capturing species in a solid-state optical measurement, accumulation of positive charges would eventually destroy the metal-sulfur bonds during prolong light exposure and long experimental measurements. Below we show that the $\lambda_{\mathrm{LSPR}}$ is fully reversible by exchanging $\mathrm{NO}_{2}-\mathrm{Ph}-\mathrm{S}$ - with $\mathrm{NH}_{2}-\mathrm{Ph}-\mathrm{S}$ - passivation and vice versa, and thus the CID phenomenon is very unlikely to have occurred under our experimental conditions. Taken together, the experimental data presented herein is a simplified approximation of the 
Drude model, which suggests the broadening of the spectral linewidth in metal nanostructures is expected to occur with an increasing $\mathrm{N}_{\mathrm{e}}$.

Finally, we experimentally measured the $\phi$ of X-Ph-S- ligand-passivated Au TNPs using ultraviolet photoelectron spectroscopy (UPS) to further examine and support our electron wave function delocalization mechanism. Figure $3 \mathrm{C}$ provides the UPS spectra of Au when TNPs were functionalized with various X-Ph-S- ligands. Additional UV-photoelectron spectra are provided in the Supporting Information (see Figure S6). The secondary electron cutoff shifts gradually to lower binding energy with electron donating X-Ph-S- ligands with respect to H-Ph-S-, while an opposite trend is observed for X-Ph-S- ligands with electron withdrawing groups. We observe an increase in the $\phi$ (Fermi level of Au moves towards more negative energy with respect to vacuum) for $\mathrm{NH}_{2}$-Ph-S-passivated Au TNPs which suggests decreasing $\mathrm{N}_{\mathrm{e}}$ in TNPs, see Figure 3D. Contrastly, $\mathrm{NO}_{2}-\mathrm{Ph}-\mathrm{S}$ - causes a decrease in the $\phi$ of $\mathrm{Au}$ indicating an increase in $\mathrm{N}_{\mathrm{e}}$. Ligand-induced changes in $\phi$ are reported in the literature for semiconductor $\mathrm{PbS}$ nanocrystals and planar metallic (Au and Ag) substrates by utilizing the UPS technique. ${ }^{33-35}$ Particularly, Beard et al. ${ }^{35}$ showed that the dipole moment of organic ligands could modulate the $\phi$ of $\mathrm{PbS}$ nanocrystals. Although we did not take the ligand dipole moment in to consideration when determined the $\phi$, but to our knowledge, we show for the first time a large change in $\phi(1.9 \mathrm{eV})$ for plasmonic nanostructures because of the variation in their surface passivating ligands. Our UPS data are in agreement with the literature demonstrating changes in redox potential ${ }^{36}$ of ultrasmall Au nanocrystals upon functionalization with X-Ph-S- ligands. Taken together, under our experimental conditions the changes in $\mathrm{N}_{\mathrm{e}}$ support our proposed model (Figure 2) that $\mathrm{X}$ Ph-S- ligands with the capability of donating electrons to metal nanostructures can reduce the $\mathrm{N}_{\mathrm{e}}$ and increase the $\phi$ of a plasmonic nanostructures under our experimental conditions. 

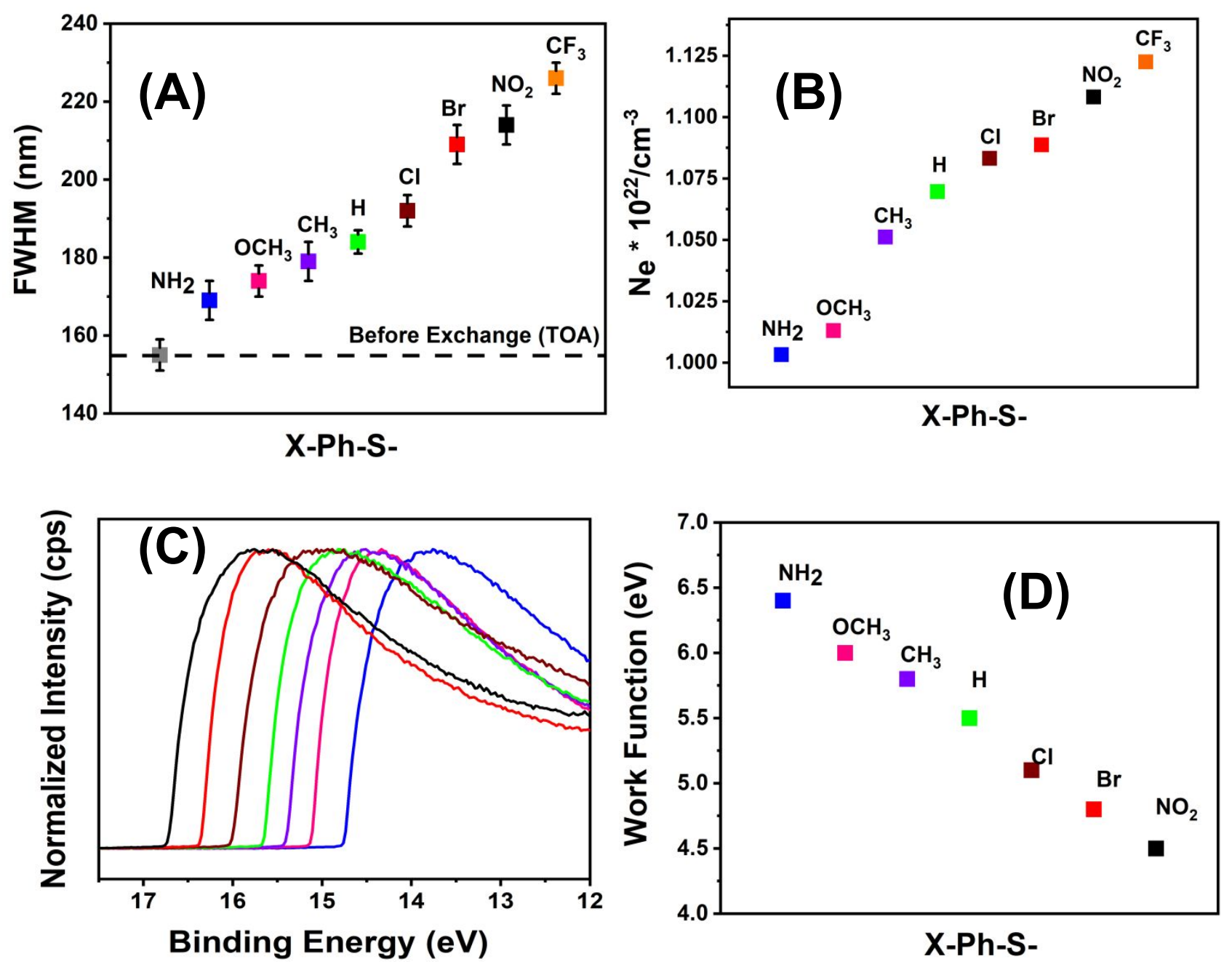

Figure 3. Quantification of ligand-controlled electronic parameters of Au TNPs. (A) Changes in the full width half maxima of the LSPR peak measured upon passivation of $A u$ TNPs with different X-Ph-S- ligands. (B) Summary of the calculated $N_{e}$ changes upon 
passivation of TNPs with X-Ph-S- ligands. Upon passivation with $\mathrm{CF}_{3}-\mathrm{Ph}-\mathrm{S}-, \mathrm{N}_{\mathrm{e}}$ increases $5.2 \%$, and $\mathrm{NH}_{2}-\mathrm{Ph}-\mathrm{S}$ - passivation leads to a decrease in $\mathrm{N}_{e}$ of $6.2 \%$ in comparison to the $\mathrm{N}_{e}$ of $\mathrm{H}-\mathrm{Ph}$ S-passivated Au TNPs. (C) Secondary electron cut-off region of UPS spectra used to determine the work function ( $\phi$ ) of X-Ph-S-passivated Au TNPs: - $\mathrm{NH}_{2}$ (blue curve), $-\mathrm{OCH}_{3}$ (pink curve), $\mathrm{CH}_{3}$ (purple curve), - $\mathrm{H}$ (green curve), - $\mathrm{Cl}$ (wine curve), - $\mathrm{Br}$ (red curve), and $-\mathrm{NO}_{2}$ (black curve). (D) Calculated $\phi$ of Au TNPs as a function of electronic properties of surface passivating ligands.

Figure 4A-C, and Figures $\mathbf{S} 7$ and 8 show the reversible tuning of the $\lambda_{\mathrm{LSPR}}$ and FWHM of $A u$ TNPs upon passivation with different X-Ph-S- ligands. Exchanging $\mathrm{H}-\mathrm{Ph}-\mathrm{S}$ - by $\mathrm{NH}_{2}-\mathrm{Ph}-\mathrm{S}$ provides a $\Delta \lambda_{\mathrm{LSPR}}$ of $+26 \mathrm{~nm}$ (red-shift), and then a $\Delta \lambda_{\mathrm{LSPR}}$ of $-40 \mathrm{~nm}$ (blue-shift) is observed when $\mathrm{NH}_{2}$-Ph-S- was replaced by $\mathrm{NO}_{2}$-Ph-S- on the surface of Au TNPs. Finally, a $\Delta \lambda_{\mathrm{LSPR}}$ of $+14 \mathrm{~nm}$ (red-shift) is detected upon exchanging $\mathrm{NO}_{2}$-Ph-S- for $\mathrm{H}-\mathrm{Ph}-\mathrm{S}$-. The exchange reactions have been performed up to five cycles without detecting any noticeable differences in the overall $\Delta \lambda_{\text {LSPR }}$ values. Fully reversible $\Delta \lambda_{\text {LSPR }}$ shifts are also achieved by exchanging $\mathrm{H}-\mathrm{Ph}-\mathrm{S}$ - with $\mathrm{NO}_{2^{-}}$ Ph-S- followed by $\mathrm{NH}_{2}$-Ph-S- (see Figure S9). In parallel, ligand exchange reactions produce reversible changes in the FWHM values, as shown in Figure 4C. The FWHM values in eV for Figure 4C is shown in Figure S10. Again, it is found that the Drude model does not follow the trends in $\Delta \lambda_{\mathrm{LSPR}}$ and FWHM values during reversible ligand exchange in our system. It is important to mention that we conducted SERS measurements utilizing the strong EM-field enhancement property of our chemically synthesized Au TNPs during the exchange reaction to confirm the complete removal of bound ligands by exchange ligands. ${ }^{37-40}$ As illustrated in Figure $\mathbf{5 A}$, the SERS intensity of the C-S stretch at $1083 \mathrm{~cm}^{-1}$ remains present in all the samples during the exchange reactions. Importantly, both the $\mathrm{C}-\mathrm{S}$ and aromatic $\mathrm{C}=\mathrm{C}\left(1573 \mathrm{~cm}^{-1}\right)$ stretches are constant in intensity throughout the exchange (see Figure 5B). This is an expected result because exchanging $\mathrm{H}-\mathrm{Ph}-\mathrm{S}$ - by $\mathrm{NH}_{2}-\mathrm{Ph}-\mathrm{S}$-, and then attaching $\mathrm{NO}_{2}-\mathrm{Ph}-\mathrm{S}$ - by $\mathrm{NH}_{2}-\mathrm{Ph}-\mathrm{S}$ exchange should not change the overall density of surface passivating ligands, the number of $\mathrm{C}-\mathrm{S}$ and aromatic $\mathrm{C}=\mathrm{C}$ bonds, and thus the SERS intensities. Furthermore, we observe disappearance of $\mathrm{N}-\mathrm{H}$ stretch at $1390 \mathrm{~cm}^{-1}$ and appearance of $\mathrm{N}-\mathrm{O}$ stretch at $1340 \mathrm{~cm}^{-1}$ when $\mathrm{NH}_{2}-\mathrm{Ph}-\mathrm{S}$ - is replaced on the surface of TNPs by $\mathrm{NO}_{2}$-Ph-S-. Additional SERS spectra are provided in the Supporting Information (see Figure S11 and 12). We should mention that a $24 \mathrm{~h}$ exchange reaction was sufficient to replace TNP surface-bound ligands with new ligands in order to achieve a stable $\lambda_{\text {LSPR }}$ position and SERS intensity (see Figure 5B), however we did not calculate the coverage through an adsorption isotherm. Although, reversible tuning of the $\lambda_{\mathrm{LSPR}}$ 
of metal nanostructures through applied electrochemical potential has been reported in the literature, ${ }^{8}$ to the best of our knowledge, this work demonstrates for the first time reversible modulation of the $\lambda_{\mathrm{LSPR}}$ of metal nanostructures in the solid-state by controlling the electronic properties of organic ligands. Depending on the chemical nature of the para-substitution, we hypothesize that the sequential ligand exchange with $\mathrm{X}-\mathrm{Ph}-\mathrm{SH}$ reestablishes HOMOs' and LUMOs' that allow electron wave functions delocalization from Au TNPs to passivating ligands and vice versa. Our $\phi$ calculations also support this hypothesis. The reversibility of $\lambda_{\mathrm{LSPR}}$ tuning also suggests that no charge transfer has taken place between TNPs and X-Ph-S-, because under such condition Au TNPs would accumulate excess electric change. Excess charge would potentially destroy the TNPS, and thus the $\lambda_{\text {LSPR }}$ position and FWHM would not be fully reversible as many cycles as we observed under our experimental conditions. 
(A)
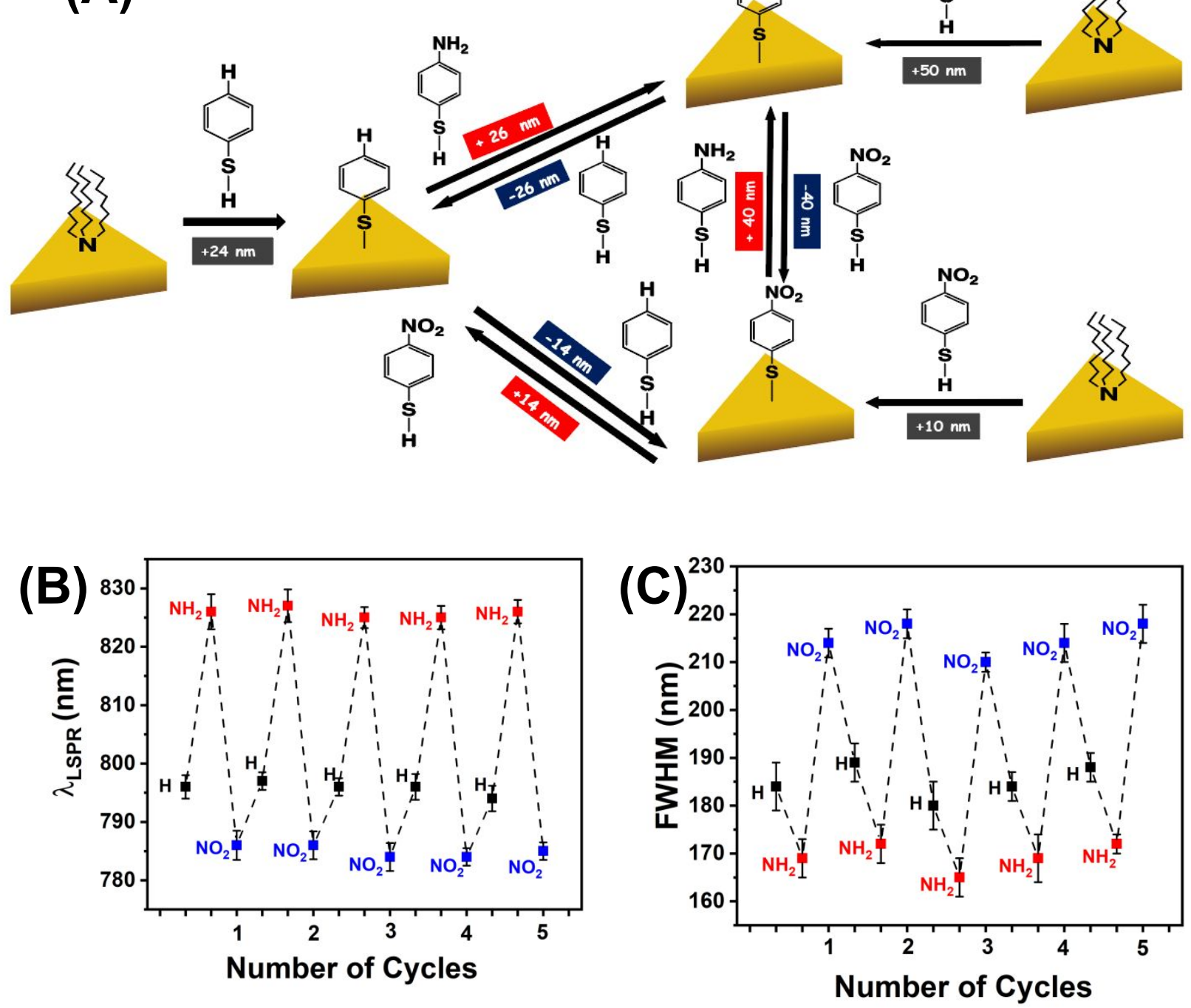

Figure 4. Ligand-controlled reversible modulation of LSPR properties of Au TNPs. (A) Schematic representation showing reversible ligand exchange reactions with various $X$-Ph-Sligands. (B) Position of the $\lambda_{\mathrm{LSPR}}$ after passivating the surface of TNPs with H-Ph-S- (black squares, $\lambda_{\mathrm{LSPR}}=\sim 796 \mathrm{~nm}$ ) and then after $\mathrm{H}$-Ph-S- was exchanged for $\mathrm{NH}_{2}-\mathrm{Ph}$-S- (red squares, $\lambda_{\mathrm{LSPR}}=\sim 828 \mathrm{~nm}$ ), which was next replaced by $\mathrm{NO}_{2}-\mathrm{Ph}$-S- (blue squares, $\lambda_{\mathrm{LSPR}}=\sim 782 \mathrm{~nm}$ ). Representative UV-visible extinction spectra are provided in the supporting Information. Reversible tuning of $\lambda_{\mathrm{LSPR}}$ position is carried out for five successive cycles. (C) Average FWHM value of the dipole peak of Au TNPs during the sequential ligand exchange with the three different-type of X-Ph-S- ligands. 

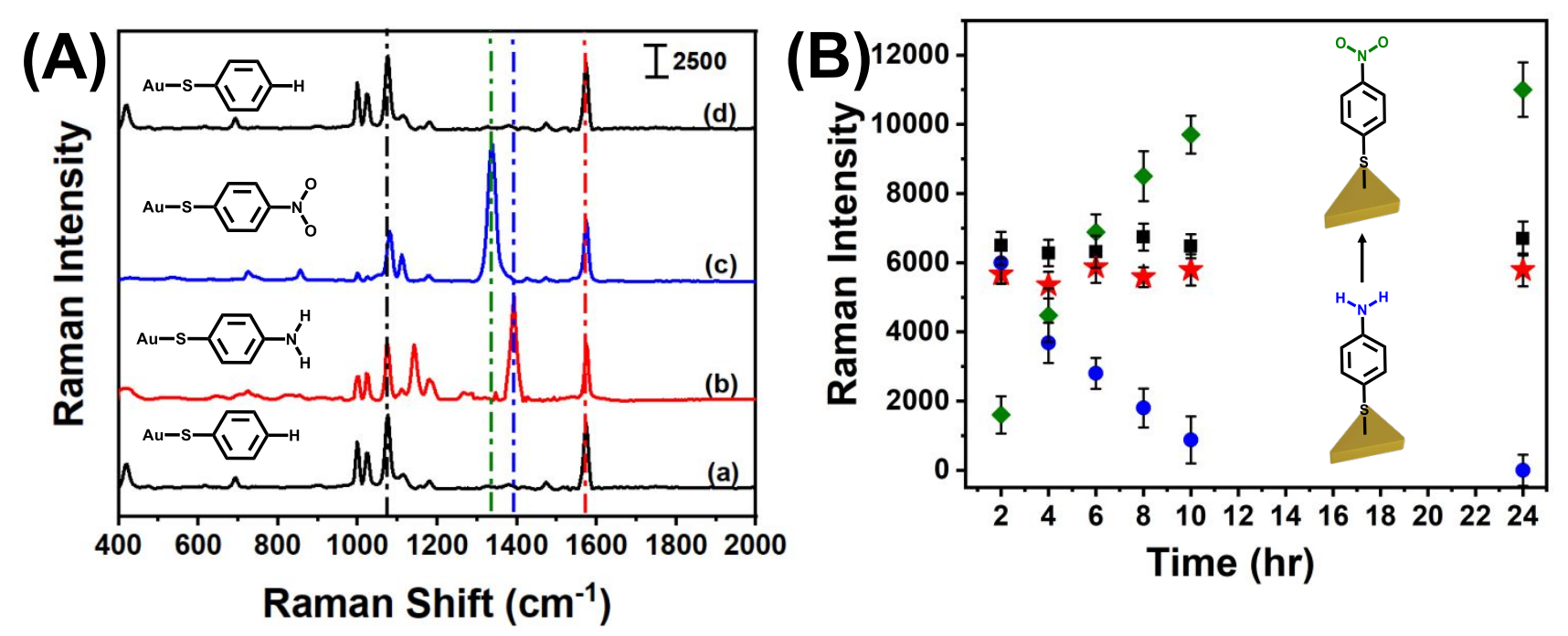

Figure 5. SERS-based Monitoring of Sequential Exchange Reactions of Au TNPs with X-

Ph-S- Ligands. (A) SERS spectrum of H-Ph-S-passivated Au TNPs (a), after exchanging H-PhS- with $\mathrm{NH}_{2}-\mathrm{Ph}$-S- ligands (b), which were next replaced by $\mathrm{NO}_{2}-\mathrm{Ph}-\mathrm{S}$ - (c), and finally $\mathrm{H}-\mathrm{Ph}-\mathrm{S}$ passivated Au TNPs were prepared by exchanging $\mathrm{NO}_{2}-\mathrm{Ph}-\mathrm{S}$ - with $\mathrm{H}-\mathrm{Ph}-\mathrm{S}-(\mathrm{d})$. Black, red, green, and blue dash lines represent $C$-S stretch at $1083 \mathrm{~cm}^{-1}$, aromatic $C=C$ stretch at 1573 $\mathrm{cm}^{-1}, \mathrm{~N}-\mathrm{O}$ stretch at $1340 \mathrm{~cm}^{-1}$ and $\mathrm{N}-\mathrm{H}$ stretch at $1390 \mathrm{~cm}^{-1}$. (B) Time dependent SERS intensity of different stretches during $24 \mathrm{hr}$ ligand exchange reactions at room temperature: $\mathrm{C}-\mathrm{S}$ (black squares), aromatic $\mathrm{C}=\mathrm{C}$ - (red stars), $\mathrm{N}-\mathrm{H}$ (blue spheres), and $\mathrm{N}-\mathrm{O}$ (green diamonds) stretches.

Finally, another possibility for the $\Delta \boldsymbol{\lambda}_{\mathrm{LSPR}}$ modulation of Au TNPs upon X-Ph-S- ligand passivation is the variable degree of ligand attachment in which the higher the number of bound ligands, larger the $\Delta \lambda_{\text {LSPR }}$ shift. One could argue that if the ligand density (number of ligand/nm²) on TNPs varies in the following order: $\mathrm{NO}_{2}-\mathrm{Ph}-\mathrm{S}->\mathrm{H}-\mathrm{Ph}-\mathrm{S}->\mathrm{NH}_{2}-\mathrm{Ph}-\mathrm{S}-$, this would provide the highest and the lowest $\Delta \lambda_{\mathrm{LSPR}}$ for $\mathrm{NH}_{2}$-Ph-S- and $\mathrm{NO}_{2}$-Ph-S-, respectively. To quantify the number of ligands attached to the TNP surface, we conducted ${ }^{1} \mathrm{H}$ NMR analysis using ferrocene as an internal standard (see Figure S13-S15) for three different thiophenolate-passivated $\mathrm{Au}$ TNPs with X-Ph-S- $\left(X=-\mathrm{NH}_{2},-\mathrm{H}\right.$, and $\left.-\mathrm{NO}_{2}\right)$ ligands. The supporting Information provides a detailed experimental procedure and quantification methods. Our results show nearly an identical surface coverage for all three X-Ph-S- (3.1-3.4 ligand/nm²). This value is very consistent with the thiolated ligand grafting density on metal nanostructures. ${ }^{41}$ Taken together, 
our unique structural construct of Au TNPs allows reversible modulation of the Au free carrier concentrations and $\lambda_{\mathrm{LSPR}}$ in which the energy level alignment between Au and ligands profoundly influences the magnitude of the $\mathrm{N}_{e}$ values.

In summary, this work capitalizes on a previously unexplored nanostructure-surface ligand construct that demonstrates reversible tuning (up to five cycles) of the plasmoelectric effect of metallic nanostructures. Our in-depth spectroscopic characterization shows that $\sim 12 \%$ of the plasmon active electrons $\mathrm{N}_{\mathrm{e}}$ of Au TNPs can be modulated through passivating their surface with X-Ph-S- ligands with electron withdrawing and donating groups. The trends in observed LSPR shifts and spectra linewidths of TNPs upon functionalization with different $\mathrm{X}$-Ph-S- ligands are opposite to the Drude model. We have found that the electron donating group $\left(-\mathrm{NH}_{2}\right)$ provides an $\sim 26 \mathrm{~nm}$ LSPR red-shift while the electron withdrawing group $\left(-\mathrm{NO}_{2}\right)$ provides an $\sim 14 \mathrm{~nm}$ blue-shift with respect to $-\mathrm{H}$ ]. Using its simplest interpretation, we have also developed orbital interaction diagrams in the solid-state to explain the LSPR properties of TNPs under our experimental conditions. We hypothesize that the energy level alignment between the continuum of states of the Au TNP and the HOMO/LUMO of X-Ph-S- produces hybrid MOs that participate in electrons wave function delocalization, which in turn reversibly alters the LSPR properties (plasmoelectric effects). Therefore, we would expect that the degree of electron wave function delocalization will vary if the strength of electron withdrawing or donating ability of the functional groups increase further. As a proof-of-concept, we investigated the plasmoelectric effect of Au TNPs by passivating their surface with a very strong electron donating group, 4(dimethylamino)thiophenol (4-DMAT) and a very strong electron withdrawing group, 3,5Bis(trifluoromethyl)benzenethiol (3,5-TFMBT). We observe a $+36 \mathrm{~nm} \lambda_{\text {LSPR }}$ red-shift and a -36 $\mathrm{nm}$ blue shift for 4-DMAT and 3,5-TFMBT, respectively, in comparison to H-Ph-S-passivated TNPs (data not shown). Importantly, the linewidth and UPS studies add additional strong support for the model. We should also mention that the MO model we have presented here has some limitations including the lack of participation of multiple HOMOs and LUMOs in the formation of hybrid MOs. Furthermore, we did not take ligand dipole moment into consideration while determining the $\mathrm{N}_{\mathrm{e}}$ and $\phi$. Further in-depth theoretical investigations are required to precisely quantify the observed LSPR properties in our Au-S-Ph-X systems. Nevertheless, the unique LSPR behavior of ligand-passivated Au TNPs could be due to their sharp tips and edges. The higher free carrier conentration of TNPs potentially reduces effective energy barrier and facilitates the delocalization of electron wave functions that together result in an unprecedentedly large change in their work function. This is the first example in which LSPR 
and UPS spectroscopic techniques have been used to monitor the plasmoelectric effect in a metallic nanostructure. Taken together, controlling charge delocalization at the metal nanostructure-organic ligand (hybrid nanoconjugate) interface should lead to discovery of unique hybrid nanoplasmonic materials in which LSPR properties of metallic nanostructures can be tuned through programmable manipulation of charge injection/withdraw by surface ligand shells. The hybrid conjugates are capable of providing both chemical versatility of organic ligands and the shape dependency of LSPR properties ${ }^{3,4}$ of metal nanostructures that are expected to expedite the fabrication of next generation nanoplasmonic devices and preparation of efficient photocatalysts. ${ }^{11-15,42}$

\title{
ASSOCIATED CONTENT
}

Supporting Information. Detailed experimental procedures for Au TNPs synthesis, ligand exchange reactions, and various spectroscopic and microscopic characterizations, and additional extinction, Raman and NMR spectra, refractive index table, and additional discussion. These materials are available free of charge.(PDF)

\author{
AUTHOR INFORMATION \\ Corresponding Author \\ *Rajesh Sardar; Email: rsardar@iupui.edu
}

\begin{abstract}
AUTHOR CONTRIBUTIONS
T.L., and R.S. designed experiments, T.L. conducted all UV-vis and Raman measurements, microscopy analysis, and analyzed data. M.N. performed DFT calculations. M.J. helped in AFM analysis. T.L., B.B.M. and R.S. interpreted the data. T.L. wrote the manuscript, and B.B.M. and R.S. edited and revised the manuscript for appropriate presentation of the findings. All authors discussed the results and contributed to the final manuscript.
\end{abstract}

\section{FUNDING}

The authors acknowledge financial support by the National Science Foundation under grant no. CBET-1604617. 


\section{ACKNOWLEDGMENT}

UPS analysis was conducted at the University of North Carolina CHANL Facility. We also thank Dr. Carrie Donley (UNC Chapel Hill) for collecting UPS spectra and providing helpful guidance with data analysis.

\section{REFERENCES}

1. Zhao, J.; Pinchuk, A. O.; McMahon, J. M.; Li, S.; Ausman, L. K.; Atkinson, A. L.; Schatz, G. C. Methods for Describing the Electromagnetic Properties of Silver and Gold Nanoparticles. Acc. Chem. Res. 2008, 41, 1710-1720.

2. Mie, G. Contributions to the Optics of Turbid Media, Especially Colloidal Metal Solutions. Ann. Phys. 1908, 330, 377-445.

3. Halas, N. J.; Lal, S.; Chang, W.-S.; Link, S.; Nordlander, P. Plasmons in Strongly Coupled Metallic Nanostructures. Chem. Rev. 2011, 111, 3913-3961.

4. Mayer, K. M.; Hafner, J. H. Localized Surface Plasmon Resonance Sensors. Chem. Rev. 2011, 111, 3828-3857.

5. Dionne, J. A. Nanoplasmonics: Plasmons rock in metal bands. Nature materials 2013, 12, 380-381.

6. Mulvaney, P. Surface Plasmon Spectroscopy of Nanosized Metal Particles. Langmuir 1996, 12, 788-800.

7. Warren, S. C.; Walker, D. A.; Grzybowski, B. A. Plasmoelectronics: Coupling Plasmonic Excitation with Electron Flow. Langmuir 2012, 28, 9093-9102.

8. Sheldon, M. T.; van de Groep, J.; Brown, A. M.; Polman, A.; Atwater, H. A. Plasmoelectric potentials in metal nanostructures. Science 2014, 346, 828-831.

9. Hoener, B. S.; Zhang, H.; Heiderscheit, T. S.; Kirchner, S. R.; De Silva Indrasekara, A. S.; Baiyasi, R.; Cai, Y.; Nordlander, P.; Link, S.; Landes, C. F., et al. Spectral Response of Plasmonic Gold Nanoparticles to Capacitive Charging: Morphology Effects. J. Phys. Chem. Lett. 2017, 8, 2681-2688.

10. Navarrete, J.; Siefe, C.; Alcantar, S.; Belt, M.; Stucky, G. D.; Moskovits, M. Merely Measuring the UV-Visible Spectrum of Gold Nanoparticles Can Change Their Charge State. Nano Lett. 2018, 18, 669-674.

11. Ueno, K.; Oshikiri, T.; Sun, Q.; Shi, X.; Misawa, H. Solid-State Plasmonic Solar Cells. Chem. Rev. 2018, 118, 2955-2993.

12. Atwater, H. A.; Polman, A. Plasmonics for improved photovoltaic devices. Nature materials 2010, 9, 205-213.

13. Pastoriza-Santos, I.; Kinnear, C.; Pérez-Juste, J.; Mulvaney, P.; Liz-Marzán, L. M. Plasmonic polymer nanocomposites. Nat. Rev. Mater. 2018, 3, 375-391.

14. Linic, S.; Christopher, P.; Ingram, D. B. Plasmonic-metal nanostructures for efficient conversion of solar to chemical energy. Nature materials 2011, 10, 911-921.

15. Zhou, L.; Swearer, D. F.; Zhang, C.; Robatjazi, H.; Zhao, H.; Henderson, L.; Dong, L.; Christopher, P.; Carter, E. A.; Nordlander, P., et al. Quantifying hot carrier and thermal contributions in plasmonic photocatalysis. Science 2018, 362, 69-72.

16. Wu, K.; Chen, J.; McBride, J. R.; Lian, T. Efficient hot-electron transfer by a plasmoninduced interfacial charge-transfer transition. Science 2015, 349, 632.

17. Joshi, G. K.; Deitz-McElyea, S.; Liyanage, T.; Lawrence, K.; Mali, S.; Sardar, R.; Korc, M. Label-Free Nanoplasmonic-Based Short Noncoding RNA Sensing at Attomolar 
Concentrations Allows for Quantitative and Highly Specific Assay of MicroRNA-10b in Biological Fluids and Circulating Exosomes. ACS Nano 2015, 9, 11075-11089.

18. Joshi, G. K.; McClory, P. J.; Dolai, S.; Sardar, R. Improved localized surface plasmon resonance biosensing sensitivity based on chemically-synthesized gold nanoprisms as plasmonic transducers. J. Mater. Chem. 2012, 22, 923-931.

19. Joshi, G. K.; Blodgett, K. N.; Muhoberac, B. B.; Johnson, M. A.; Smith, K. A.; Sardar, R. Ultrasensitive Photoreversible Molecular Sensors of Azobenzene-Functionalized Plasmonic Nanoantennas. Nano Lett. 2014, 14, 532-540.

20. Lee, S. Y.; Tsalu, P. V.; Kim, G. W.; Seo, M. J.; Hong, J. W.; Ha, J. W. Tuning chemical interface damping: Interfacial electronic effects of adsorbate molecules and sharp tips of single gold bipyramids. Nano Lett. 2019, 19, 2568-2574.

21. Liyanage, T.; Masterson, A. N.; Oyem, H. H.; Kaimakliotis, H.; Nguyen, H.; Sardar, R. Plasmoelectronic-Based Ultrasensitive Assay of Tumor Suppressor microRNAs Directly in Patient Plasma: Design of Highly Specific Early Cancer Diagnostic Technology. Anal. Chem. 2019, 91, 1894-1903.

22. Joshi, G. K.; Deitz-McElyea, S.; Johnson, M.; Mali, S.; Korc, M.; Sardar, R. Highly Specific Plasmonic Biosensors for Ultrasensitive MicroRNA Detection in Plasma from Pancreatic Cancer Patients. Nano Lett. 2014, 14, 6955-6963.

23. Joshi, G. K.; McClory, P. J.; Muhoberac, B. B.; Kumbhar, A.; Smith, K. A.; Sardar, R. Designing Efficient Localized Surface Plasmon Resonance-Based Sensing Platforms: Optimization of Sensor Response by Controlling the Edge Length of Gold Nanoprisms. $J$. Phys. Chem. C 2012, 116, 20990-21000.

24. Yu, S.; Wilson, A. J.; Heo, J.; Jain, P. K. Plasmonic Control of Multi-Electron Transfer and $\mathrm{C}-\mathrm{C}$ Coupling in Visible-Light-Driven $\mathrm{CO} 2$ Reduction on Au Nanoparticles. Nano Lett. 2018, 18, 2189-2194.

25. Hoffmann, R. A chemical and theoretical way to look at bonding on surfaces. Rev. Mod. Phys. 1988, 60, 601-628.

26. Govorov, A. O.; Zhang, H.; Demir, H. V.; Gun'ko, Y. K. Photogeneration of hot plasmonic electrons with metal nanocrystals: Quantum description and potential applications. Nano Today 2014, 9, 85-101.

27. Zhu, M.; Aikens, C. M.; Hollander, F. J.; Schatz, G. C.; Jin, R. Correlating the Crystal Structure of A Thiol-Protected Au25 Cluster and Optical Properties. J. Am. Chem. Soc. 2008, 130, 5883-5885.

28. Sardar, R.; Funston, A. M.; Mulvaney, P.; Murray, R. W. Gold Nanoparticles: Past, Present, and Future. Langmuir 2009, 25, 13840-13851.

29. Jadzinsky, P. D.; Calero, G.; Ackerson, C. J.; Bushnell, D. A.; Kornberg, R. D. Structure of a Thiol Monolayer-Protected Gold Nanoparticle at $1.1 \AA$ Resolution. Science 2007, 318, 430-433.

30. Foerster, B.; Joplin, A.; Kaefer, K.; Celiksoy, S.; Link, S.; Sönnichsen, C. Chemical Interface Damping Depends on Electrons Reaching the Surface. ACS Nano 2017, 11, 2886-2893.

31. Luther, J. M.; Jain, P. K.; Ewers, T.; Alivisatos, A. P. Localized surface plasmon resonances arising from free carriers in doped quantum dots. Nature materials 2011, 10, 361-366.

32. Hoggard, A.; Wang, L.-Y.; Ma, L.; Fang, Y.; You, G.; Olson, J.; Liu, Z.; Chang, W.-S.; Ajayan, P. M.; Link, S. Using the Plasmon Linewidth To Calculate the Time and 
Efficiency of Electron Transfer between Gold Nanorods and Graphene. ACS Nano 2013, 7, 11209-11217.

33. Brown, P. R.; Kim, D.; Lunt, R. R.; Zhao, N.; Bawendi, M. G.; Grossman, J. C.; Bulović, V. Energy Level Modification in Lead Sulfide Quantum Dot Thin Films through Ligand Exchange. ACS Nano 2014, 8, 5863-5872.

34. Alloway, D. M.; Graham, A. L.; Yang, X.; Mudalige, A.; Colorado, R.; Wysocki, V. H.; Pemberton, J. E.; Lee, T. R.; Wysocki, R. J.; Armstrong, N. R. Tuning the Effective Work Function of Gold and Silver Using $\omega$-Functionalized Alkanethiols: Varying Surface Composition through Dilution and Choice of Terminal Groups. J. Phys. Chem. C 2009, 113, 20328-20334.

35. Kroupa, D. M.; Voros, M.; Brawand, N. P.; McNichols, B. W.; Miller, E. M.; Gu, J.; Nozik, A. J.; Sellinger, A.; Galli, G.; Beard, M. C. Tuning colloidal quantum dot band edge positions through solution-phase surface chemistry modification. Nat. Commun. 2017, 8, 15257.

36. Guo, R.; Murray, R. W. Substituent Effects on Redox Potentials and Optical Gap Energies of Molecule-like Au38(SPhX)24 Nanoparticles. J. Am. Chem. Soc. 2005, 127, 12140-12143.

37. Scarabelli, L.; Coronado-Puchau, M.; Giner-Casares, J. J.; Langer, J.; Liz-Marzán, L. M. Monodisperse Gold Nanotriangles: Size Control, Large-Scale Self-Assembly, and Performance in Surface-Enhanced Raman Scattering. ACS Nano 2014, 8, 5833-5842.

38. Liyanage, T.; Rael, A.; Shaffer, S.; Zaidi, S.; Goodpaster, J. V.; Sardar, R. Fabrication of a self-assembled and flexible SERS nanosensor for explosive detection at parts-perquadrillion levels from fingerprints. Analyst 2018, 143, 2012-2022.

39. Joshi, G. K.; White, S. L.; Johnson, M. A.; Sardar, R.; Jain, P. K. Ultrashort, AngstromScale Decay of Surface-Enhanced Raman Scattering at Hot Spots. J. Phys. Chem. C 2016, 120, 24973-24981.

40. Kim, J.; Song, X.; Ji, F.; Luo, B.; Ice, N. F.; Liu, Q.; Zhang, Q.; Chen, Q. Polymorphic Assembly from Beveled Gold Triangular Nanoprisms. Nano Lett. 2017, 17, 3270-3275.

41. Goldmann, C.; Lazzari, R.; Paquez, X.; Boissiere, C.; Ribot, F.; Sanchez, C.; Chaneac, C.; Portehault, D. Charge Transfer at Hybrid Interfaces: Plasmonics of Aromatic ThiolCapped Gold Nanoparticles. ACS Nano 2015, 9, 7572-7582.

42. Knight, M. W.; Sobhani, H.; Nordlander, P.; Halas, N. J. Photodetection with Active Optical Antennas. Science 2011, 332, 702-704. 